\title{
Silent Consequences of COVID-19: Why It's Critical to Recover Routine Vaccination Rates Through Equitable Vaccine Policies and Practices
}

\author{
Ava Skolnik, MPH \\ Alexandra Bhatti, JD, MPH \\ Anna Larson, MPH \\ Rachel Mitrovich, DrPH, MPH \\ Merck \& Co, Inc, Kenilworth, New Jersey
}

\begin{abstract}
In the United States, routine vaccination rates have plummeted across all age groups due to the COVID-19 pandemic, with our most vulnerable and underserved populations suffering the greatest declines. Returning to a "new normal" and recovering our nation's health and economy is of the utmost importance; however, there is a critical need to recover and protect communities against the spread of other vaccine-preventable diseases and outbreaks. While routine vaccination rates are slowly recovering for certain age groups, the introduction of COVID-19 vaccines adds complexities and challenges to recovery efforts. If not addressed, hard-won gains in routine vaccination may be lost, which could result in communities missing out on the social, economic, and health benefits offered by vaccinations.

There is an urgent need to utilize evidence-based and innovative strategies to support both immediate and long-term efforts to recover, maintain, and sustain routine vaccination. Key short-term strategies include leveraging digital and mainstream media to drive awareness, coordinating across health and education sectors, utilizing centralized reminder recall, expanding access points to vaccination services, and elevating trusted voices for vaccination. In order to build back stronger, long-term strategies include enhancing immunization information systems, mitigating financial barriers to vaccination, investing in building vaccine confidence, and ensuring sustainable funding for immunization infrastructure.
\end{abstract}

Ann Fam Med 2021;19:527-531. https://doi.org/10.1370/afm.2730.

Annals "Online First" article.

\section{INTRODUCTION}

\section{The COVID-19 Pandemic is a Critical Reminder of the Importance of Vaccination ${ }^{1}$}

$T_{0}$ help end the COVID-19 pandemic, individuals across the United States, from government officials to frontline workers, are engaged in a tireless effort to ensure Americans have access to and are vaccinated against COVID $-19 .{ }^{2}$ Ending the pandemic is mission critical ${ }_{i}$ however, it is also essential to build back stronger by developing and implementing measures to recover from subsequent public health consequences of the pandemic.

One such consequence is the severe disruption to routine vaccination services, resulting in considerable deficits in vaccination rates across all age groups. ${ }^{1,3,4}$ Vaccination is a global health and development success story. ${ }^{4}$ In the United States alone, routine childhood vaccination has been estimated to prevent approximately 42,000 deaths and 20 million cases of disease, averting an estimated $\$ 76$ billion in total societal costs-in a single birth cohort alone. ${ }^{5}$

Disruptions to routine vaccination services have been recorded throughout the pandemic. Private claims data from 3 routine childhood vaccines, measles-mumps-rubella (MMR), diphtheria, tetanus, and acellular 
pertussis $(\mathrm{DTaP})$, and polio suggest that an estimated 9 million doses may have been missed in 2020-and up to a $26 \%$ drop in those 3 vaccines between January and September of last year. ${ }^{6}$ An analysis from 10 state immunization information systems (IIS) estimates more than a $60 \%$ decline in MMR for children aged 2 to 8 years, and over a $60 \%$ decline in human papillomavirus (HPV) vaccination for adolescents aged 9 to 12 years from March-May of 2020 compared with MarchMay of $2019 .^{7}$ These disruptions are attributable, in part, to social distancing measures, reallocation of health care workers, de-prioritization of routine health care services, and concerns that patients and caregivers seeking routine preventive services, like vaccinations, may be exposed to COVID-19., ${ }^{8,9}$

Local accounts from across the United States further demonstrate the magnitude of impact. Reports from New York City indicated over a 90\% drop in vaccine doses given to children over 2 years of age between March and May of 2020..$^{10}$ Colorado experienced similar troubling trends with a decline in vaccination rates of $31 \%$ for individuals aged under 2 years, $78 \%$ for individuals aged 3 to 9 years, and $82 \%$ for individuals aged 10 to 17 years between January 2020 and May 2020." These significant declines in vaccine coverage rates (VCRs) put our communities at risk for additional vaccine-preventable disease outbreaks and, in the case of HPV vaccination, additional cases of HPV-related cancers later in life, further straining a health system already fatigued from managing the COVID-19 pandemic.

The COVID-19 pandemic has also exacerbated existing health disparities and highlighted the need to focus on vaccine equity. When examining the decline of routine vaccination services, the consequences of the pandemic have had a disparate impact on underserved populations. Public childhood non-influenza vaccine doses were down by 11.2 million at the start of 2021. ${ }^{12}$ Data from the Vaccines for Children program, serving primarily children who are insured through Medicaid, uninsured, and underinsured, reported declines for HPV vaccination (21\%), tetanus, diphtheria and acellular pertussis (Tdap) vaccination (22\%), and meningococcal vaccination (18\%) for 2020-2021 as compared with 2019.12 Traditionally underserved populations, such as those who are insured through Medicaid, have not only seen the greatest decline in routine vaccination rates, but are also are recovering at a slower rate compared with those with private insurance. ${ }^{13}$

\section{Examples of Promising Efforts Underway to Support Routine Vaccination}

The road to recovering routine vaccination rates requires cross-sectoral, concerted efforts to raise awareness of the importance of vaccination and to develop strategies to improve access to services. To address these challenges, actors across multiple sectors have taken action to address the declines in routine vaccination rates by implementing promising efforts to meet community health needs.

To raise awareness of the declines in routine vaccination, local and state health departments, Medicaid programs, commercial health plans, medical societies, nongovernmental organizations, the federal government, faith-based communities, community-based organizations, and others have implemented different strategies. ${ }^{7,14-19}$ "Calls to action" have been issued by leaders within the public health, cancer, and education communities. This includes an urgent action statement from the National Cancer Institute (NCI) that highlighted concerning declines in HPV vaccination coverage rates. The Centers for Disease Control and Prevention (CDC) released guidance about the importance of maintaining routine vaccination during the pandemic, emphasizing that vaccination services are essential health services that should be continued across the life course. ${ }^{3,20}$ The CDC's Director of the National Center for Immunization and Respiratory Diseases (NCIRD) even issued a call to action urging all stakeholders to focus efforts on reversing the collapse in adolescent vaccination. ${ }^{3}$

National organizations, such as the American Academy of Pediatrics, launched campaigns to remind patients and families of the importance of returning to their health care professionals for routine vaccinations. ${ }^{21}$ Similarly, states are encouraging patients and families to receive routine vaccinations through locally tailored campaigns, such as the Michigan Department of Public Health's "Be A Hero-VACCINATE" campaign, California's "Don't Wait Vaccinate" campaign, and the "Vax to School" campaign in Washington State. ${ }^{22-24}$

\section{Enabling Equitable Access to Vaccination Services is Key to Recovering Routine Vaccination Rates}

The COVID-19 pandemic has accelerated the adoption of telehealth options for select health services, which has changed how vaccination is recommended. Several state Medicaid programs released guidance for telehealth services recommending that health care professionals inform beneficiaries of any vaccinations that would normally be administered and encourage patients to schedule a time to administer routine vaccinations in person. ${ }^{25-27} \mathrm{~W}$ ith the increase of telehealth, however, certain populations are at risk for being left behind due lack of access to the Internet and digital illiteracy, highlighting the need for multiple care delivery models. ${ }^{28}$ 
Local hospitals and health systems have implemented innovative care delivery models to recover routine vaccination rates, such as mobile clinic vans and drive-through vaccination options. ${ }^{29,30}$ For example, Walter Reed National Military Medical Center was the beta site for innovative drive-through vaccination clinics. ${ }^{31}$ As innovative models try to meet patients where they are, practices deployed for COVID-19 vaccination should be considered for routine vaccination too. Partnerships have emerged between large health systems and community health organizations to build vaccine confidence and provide vaccination services at a convenient time and accessible location for COVID19 vaccinations, leading to success in delivering vaccinations to traditionally underserved populations. ${ }^{32}$ Models of community-academic partnerships can be expanded and replicated for routine vaccination services to ensure vaccine equity in recovery efforts. ${ }^{33}$

Federal agencies have also taken action to increase awareness and expand access to routine vaccination and address vaccine confidence. ${ }^{34}$ Recently, the Biden administration made an investment in the vaccine ecosystem by dedicating $\$ 1$ billion to build vaccine confidence, support vaccine education, improve vaccination rates for COVID-19 vaccines, as well as other vaccine preventable diseases, and support recovery of routine vaccination rates. ${ }^{35}$ The administration also made an additional $\$ 6$ billion investment in community health centers to expand access to COVID-19 vaccines in underserved communities - in part through addressing vaccine hesitancy. Although directed toward COVID19 vaccination, this infusion of funds has the potential to provide infrastructure support that could benefit the broader vaccine ecosystem. In addition, $\$ 500$ million has been allocated to support school nurses for COVID-19 vaccination efforts as COVID-19 vaccination eligibility expands to younger populations. ${ }^{36}$ These federal actions are first steps to recover vaccination rates, but vaccination rates did not reach all target goals before the pandemic. Sustained investment in the vaccine ecosystem and health infrastructure is necessary to not only recover routine vaccination rates, but also improve them.

\section{Short- and Long-Term Measures to Recover Vaccination Rates}

Even with these and other promising efforts underway, recovering from the decline in routine vaccination rates caused by the pandemic will take years. ${ }^{3}$ This recovery requires all stakeholders to drive awareness of the importance of routinely recommended vaccinations, facilitate access to vaccination, and build vaccine confidence.

There are measures that can be taken now to support recovery in the short term as well as actions that can be taken to help achieve and sustain high vaccination coverage rates, build a more resilient vaccine infrastructure, and build back stronger.

In the short term, taking immediate action to recover routine vaccination coverage rates is necessary to help mitigate the future effects of the pandemic. Short-term strategies that should be implemented now include:

- Leverage digital and mainstream media to drive awareness $^{37}$

- Share information via e-mails, newsletters, and social media from trusted messengers detailing the importance of protection from vaccine-preventable diseases

- Coordinate across health and education sectors ${ }^{3,38}$ - For populations like adolescents, who are currently eligible to be vaccinated for COVID-19, thoughtful planning is essential to ensure that they receive both COVID-19 vaccination as well as their routine and catch-up vaccinations

- Partner and coordinate with school leadership and the education community to promote the importance of vaccination to support schools remaining open and/or opening safely

- Use every opportunity to educate about routine vaccinations ${ }^{39}$

- Clinicians and health systems should use every opportunity to educate parents, caregivers, and patients about the importance of routine vaccinations, including when patients are administered a COVID-19 vaccine. This includes scheduling vaccination visits and providing a high-quality recommendation.

- Utilize centralized reminder recall ${ }^{40}$

- Communicate with state public health officials and medical societies about utilizing centralized reminder recall systems to send e-mails, text messages, patient app notifications, and mailings to patients for routinely recommended and catch-up vaccinations in an effort to bring individuals in for their vaccinations now

- Elevate trusted voices for vaccination ${ }^{33,38,41}$

- Identify organizations, parents, and youth voices in the community to highlight the drop in routine vaccination and highlight the need to return to care

- Expand access points to vaccination services to help "meet people where they are" "3, $^{38}$

- Plan vaccination sites at schools, drive-through clinics, and mobile vaccination clinics

While short-term strategies are necessary to mitigate effects now, long-term systems-strengthening measures must also be implemented to develop a resilient immunization ecosystem. Long-term strategies that may help with systems strengthening include: 
- Enhancement of immunization information systems ${ }^{42}$ - Strengthen IIS by (1) ensuring timely and complete exchange of data; (2) enabling IIS to collect data across the lifespan; and (3) dedicating sustainable financing to support IIS maintenance and enhancement

- Mitigate barriers to vaccination ${ }^{43}$

- Ensure policies, both at the federal and state level, support access to vaccination services. This includes mitigating direct costs to the patient such as eliminating out-of-pocket costs for vaccination services, as well as indirect costs to the patient such as providing transportation reimbursement for medical services such as vaccination.

- Secure sustainable funding for immunization

infrastructure

- Identify and address inefficiencies in vaccination financing and use of resources, while exploring innovative policies to secure financing for sustainable, resilient, vaccination programs

- Invest in building vaccine confidence ${ }^{33}$

- Build a strong understanding of the underlying drivers for vaccine hesitancy and strengthen local capacity to manage issues related to vaccine hesitancy while building trust in science, public health, and health care professionals

\section{CONCLUSION}

As the country continues efforts to end the COVID19 pandemic, including through COVID-19 vaccination, it is critical to remain vigilant in efforts to support routine vaccination. Routine vaccination programs require both immediate actions to recover coverage rates, as well as long-term efforts to build back stronger and protect communities from vaccinepreventable diseases in the future. Recovering vaccination rates will continue to necessitate cross-sectoral collaboration to address the COVID-19 pandemic while ensuring individuals remain up to date with CDC-recommended vaccinations.

To read or post commentaries in response to this article, see it online at https://doi.org/10.1370/afm.2730.

Key words: COVID-19; vaccine; vaccination; immunization; vaccine ecosystem; recovery; health policy; health equity; child health; adolescent health; vaccine equity

Submitted April 9, 2021; submitted, revised, June 18, 2021; accepted June 28, 2021.

\section{References}

1. Santoli JM, Lindley MC, DeSilva MB, et al. Effects of the COVID-19 pandemic on routine pediatric vaccine ordering and administration - United States, 2020. MMWR Morb Mortal Wkly Rep. 2020;69(19): 591-593. 10.15585/mmwr.mm6919e2
2. US Department of Health $\&$ Human Services. COVID-19 vaccines. Accessed Mar 23, 2021. https://www.hhs.gov/coronavirus/covid-19vaccines/index.html

3. Messonnier N. Help kids' safe return to school - get caught up on recommended vaccines. Centers for Disease Control and Prevention. Published Mar 2021. Accessed Jun 10, 2021. https://www.cdc.gov/ vaccines/hcp/clinical-resources/downloads/safe-return-school.pdf

4. National Foundation for Infectious Diseases (NFID). Issue brief: the impact of COVID-19 on US vaccination rates. Published Aug 2020. Accessed Mar 23, 2021. https://www.nfid.org/keep-up-the-rates/ issue-brief-the-impact-of-covid-19-on-us-vaccination-rates/

5. Zhou F, Shefer A, Wenger J, et al. Economic evaluation of the routine childhood immunization program in the United States, 2009. Pediatrics. 2014;133(4):577-585. 10.1542/peds.2013-0698

6. BlueCross BlueShield. Missing vaccinations during COVID-19 puts our children $\&$ communities at risk. Published 2020. Accessed Mar 23, 2021. https://www.bcbs.com/the-health-of-america/infographics/ missing-vaccinations-during-covid-19-puts-our-children-andcommunities-at-risk

7. Patel B, Murthy, Zell E, et al. Impact of the COVID-19 pandemic on administration of selected routine childhood and adolescent vaccinations-10 U.S. jurisdictions, March-September 2020. MMWR Morb Mortal Wkly Rep. 2021;70:840-845. 10.15585/mmwr. $\mathrm{mm} 7023 \mathrm{a} 2$

8. Czeisler ME, Marynak K, Clarke KEN, et al. Delay or avoidance of medical care because of COVID- 19-related concerns - United States, June 2020. MMWR Morb Mortal W/kly Rep. 2020;69(36):12501257. $10.15585 / \mathrm{mmwr} . \mathrm{mm} 6936 \mathrm{a} 4$

9. Centers for Disease Control and Prevention. Immunization works May 2020. Published May 29, 2020. Accessed Mar 23, 2021. https://www.cdc.gov/vaccines/news/news/trs/imwrks/2020/2020-05. html

10. Shapiro E. Child vaccinations plummet 63 percent, a new hurdle for NYC schools. New York Times. May 21, 2020:A16. Updated Jul 1, 2020. Accessed Mar 23, 2021. https://www.nytimes. com/2020/05/20/nyregion/coronavirus-schools-vaccinations.html

11. O'Leary ST, Trefren L, Roth H, Moss A, Severson R, Kempe A. Number of childhood and adolescent vaccinations administered before and after the COVID-19 outbreak in Colorado. JAMA Pediatr. 2021; 175(3):305-307. 10.1001/jamapediatrics.2020.4733

12. National HPV Vaccination Roundtable. Catch up now: an urgent action call for health plans to close the adolescent vaccination care gap. https://hpvroundtable.org/wp-content/uploads/2021/03/ Spring-2021-HS-Call-to-Action_FINAL.pdf

13. Mehrotra A, Chernew M, Linetsky D, Hatch H, Cutler D, Schneider E. The impact of the COVID-19 pandemic on outpatient visits: changing patterns of care in the newest COVID-19 hot spots. Commonwealth Fund. Published Aug 13, 2020. https://www. commonwealthfund.org/publications/2020/aug/impact-covid19-pandemic-outpatient-visits-changing-patterns-care-newest

14. Department of Public Health, City of Philadelphia. COVID-19 vaccine program. Accessed Mar 23, 2021. https://vax.phila.gov/index. php/covid-19/

15. Bramer CA, Kimmins LM, Swanson R, et al. Decline in child vaccination coverage during the COVID-19 pandemic - Michigan Care Improvement Registry, May 2016-May 2020. MMWR Morb Mortal Wkly Rep. 2020;69(20):630-631. 10.15585/mmwr.mm6920e1

16. California Medi-Cal Drug Use Review (DUR). 2020 immunization updates: vaccination during COVID-19, flue, HepA, and Tdap. Published Sep 30, 2020. Accessed Mar23, 2021. https://files.medi-cal. ca.gov/pubsdoco/dur/Articles/dured_30685.pdf

17. Jenco M. AAP urges vaccination as rates drop due to COVID19. American Academy of Pediatrics (AAP). Published May 8, 2020. Accessed Mar 23, 2021. https://www.aappublications.org/ news/2020/05/08/covid19vaccinations050820 
18. Korioth T. Pediatricians discuss ways to prepare ahead of COVID-19 vaccination of children. American Academy of Pediatrics (AAP). Published Dec 21, 2020. Accessed Mar 23, 2021. https://www.aap publications.org/news/2020/12/21/townhall12212020

19. Sharpe-Scott K. Report from the field: the impact of COVID-19 on local health department immunization programs. National Association of County and City Health Officials (NACCHO). Published Jun 26, 2020. Accessed Mar 23, 2021. https://www.naccho.org/blog/ articles/report-from-the-field-the-impact-of-covid-19-on-local-healthdepartment-immunization-programs

20. Centers for Disease Control and Prevention (CDC). Interim guidance for routine and influenza immunization services during the COVID-19 pandemic. Updated Apr 6, 2021. Accessed Mar 23, 2021. https://www.cdc.gov/vaccines/pandemic-guidance/index.html

21. American Academy of Pediatrics (AAP). \#CallYourPediatrician. Last updated Dec 10, 2020. Accessed Mar 23, 2021. https://services.aap. org/en/news-room/campaigns-and-toolkits/call-your-pediatrician/

22. Michigan Department of Health \& Human Services (MDHHS). Immunization resources for providers during the COVID-19 pandemic. Accessed Mar 23, 2021. https://www.michigan.gov/mdhhs/ 0,5885,7-339-73971_4911_4914-530653--,00.html

23. California Immunization Coalition. Don't wait vaccinate campaign. Accessed Mar 23, 2021. https://www.immunizeca.org/dontwait vaccinate/

24. Immunity Community. COVID-19 and immunization. Accessed Mar 23, 2021. https://immunitycommunitywa.org/covid-19-andimmunizations/

25. South Carolina Department of Health and Human Services. Medicaid bulletin: Coronavirus disease 2019 (COVID-19) temporary telephonic and telehealth services updates. Healthy Connections. Published Mar 19, 2020. Accessed Mar 23, 2021. https://msp. scdhhs.gov/covid19/sites/default/files/press-release/files/\%28202003-19\%29\%20COVID_TH\%20Bulletin\%20\%281\%29.pdf

26. North Carolina Department of Health and Human Services (NCDHHS). Special bulletin COVID-19 \#66: telehealth and virtual patient communications clinical policy modifications - well child visits. Published Apr 24, 2020. Accessed Mar 23, 2021. https:// medicaid.ncdhhs.gov/blog/2020/04/24/special-bulletin-covid-19-66telehealth-and-virtual-patient-communications-clinical

27. South Carolina Department of Health and Human Services. COVID19 telehealth policy update to well-visit coverage. Healthy Connections. Published Apr 16, 2020. Accessed Mar 23, 2021. https:// www.scdhhs.gov/press-release/coronavirus-disease-2019-covid19-update-temporary-telehealth-coverage-changes-0

28. Cantor J, McBain R, Pera M, et al. Who is (and is not) receiving telemedicine care during the COVID-19 pandemic [published online ahead of print March 6, 2021]. Am J Prev Med. 10.1016/j. amepre.2021.01.030

29. Bailey M. Mobile medicine: how a pediatric vaccination van has upended the idea of care. Published Jun 9, 2020. Accessed Mar 23, 2021. https://healthcity.bmc.org/population-health/ how-pediatric-vaccination-van-has-upended-idea-care

30. Gums J. How drive-thru vaccinations can help \#StopTheSpread. National Foundation for Infectious Diseases (NFID). Published Dec 10, 2020. Accessed Mar 23, 2021. https://www.nfid.org/2020/12/10/ how-drive-thru-vaccinations-can-help-stopthespread/
31. Little BS. Defending the homeland: WRNMMC operates drive-up immunization clinic. Health.mil. Published Jun 3, 2020. Accessed Mar 23, 2021. https://www.health.mil/News/Articles/2020/06/03/ WRNMMC-Operates-Drive-Up-Immunization-Clinic-during-COVID19-Pandemic

32. Lee $\mathrm{K}$, et al. Commentary: Operationalizing equity: a rapid-cycle innovation approach to Covid-19 vaccination in black neighborhoods. NEJM Catalyst. Published Apr 7, 2021. https://catalyst.nejm. org/doi/full/10.1056/CAT.21.0094

33. American Psychological Association. Building vaccine confidence through community engagement. Accessed Jun 10, 2021. https:// www.apa.org/topics/covid-19/equity-resources/building-vaccineconfidence.pdf

34. US Department of Health $\&$ Human Services. Catch-up to get ahead toolkit. HHS.gov. Accessed Jun 10, 2021. https://www.hhs.gov/ immunization/catch-up/index.html

35. House Committee on the Budget. American Rescue Plan Act of 2021. https://budget.house.gov/american-rescue-plan-act-2021-hr1319

36. The White House. Fact sheet: Biden-Harris administration to invest $\$ 7$ billion from American Rescue Plan to hire and train public health workers in response to COVID-19. Published May 13, 2021. Accessed Jun 10, 2021. https://www.whitehouse.gov/briefingroom/statements-releases/2021/05/13/fact-sheet-biden-harrisadministration-to-invest-7-billion-from-american-rescue-plan-to-hireand-train-public-health-workers-in-response-to-covid-19/

37. Bonnevie E, Rosenberg SD, Kummeth C, Goldbarg J, Wartella E, Smyser J. Using social media influencers to increase knowledge and positive attitudes toward the flu vaccine. PLoS One. 2020;15(10): e0240828. 10.1371/journal.pone.0240828

38. Chicago Department of Public Health. Vaccines for children news bulletin: issue number 2. Published Mar 23, 2021. Accessed Jun 10, 2021. https://www.chicagohan.org/

39. Centers for Disease Control and Prevention (CDC). Standards for practice: vaccine administration \& referral. Reviewed May 2, 2016. Accessed Jun 10, 2021. https://www.cdc.gov/vaccines/hcp/adults/ for-practice/standards/referral.html

40. Niccolai LM, Hansen CE. Practice- and community-based interventions to increase human papillomavirus vaccine coverage, a systematic review. JAMA Pediatr. 2015;169(7):686-692. 10.1001/ jamapediatrics.2015.0310

41. Association of State and Territorial Health Officials (ASTHO). Communicating effectively about vaccines: new communication resources for health officials. Published 2010. Accessed Jun 10, 2021. https://www.astho.org/Programs/Immunization/ Communicating-Effectively-About-Vaccines--New-CommunicationResources-for-Health-Officials/

42. Centers for Disease Control and Prevention (CDC). Immunization information systems (IIS). https://www.cdc.gov/vaccines/programs/ iis/index.html

43. Stoecker C, Stewart AM, Lindley MC. The cost of cost-sharing: the impact of Medicaid benefit design on influenza vaccination uptake. Vaccines (Basel). 2017;5(1):1-8. 10.3390/vaccines5010008 